\title{
The Modified Palomba Economic Model by Difference Equations and its Stability Analysis
}

\author{
Bahatdin Daşbaşı \\ Kayseri University, Faculty of Engineering, Architecture and Design, Department of Engineering Basic Sciences, \\ 38280, Kayseri/Turkey \\ ${ }^{*}$ E-mail address: bdasbasi@kayseri.edu.tr
}

ORCID numbers of authors: 0000-0001-8201-7495

Received date: 13.07.2021

Accepted date: 17.08 .2021

\begin{abstract}
In this study, Palomba economic model was analyzed through difference equations. For these products given as capital and consumer goods in the model, their non-marketable loss rates were also taken into account. Tirivial and non-tirivial equilibrium points were found and local asymptotic stability (LAS) conditions of these equilibrium points were investigated. Although the non-trivial equilibrium point is always unstable, the stability conditions of the trivial equilibrium point were found. In this way, in addition to the thought put forward by Palomba, the absence of periodicity was expressed too. The findings were supported by numerical studies.
\end{abstract}

Keywords: Palomba Economic Model, Difference Equation System, Stability Analysis.

\section{Introduction}

Both engineers, naturalists and researchers and practitioners working in the economic and social sciences use mathematical models of the systems, in which they study. These models, which are expected to be solved in any way, or which are predicted to be solved under certain conditions, and which are expressed through mathematical equations, give a simplified description of real-life problems [1]. It is seen that this modeling process is mostly done by using differential equations and difference equations in the literature. The application areas of these equations are widely used in the mathematical modeling studies in fields such as finance, accounting and economics in social sciences.

The difference equation theory has been greatly developed over the last three hundred years. Generally, the difference equations describe the change in a variable between two periods. By using these equations, the relevant factors that cause changes in the values of functions in different time periods can be examined [2].

Mathematical models are widely used in economics to represent relationships between various quantities such as price, level of production, demand, employment and investment [3]. Palomba (1939) considered an economy in which only two types of goods (products) existed as capital and consumer goods. Palomba's proposed model has the following assumptions [4]: 
- Two types of goods as $a$ and $b$ exist. Type- $a$ goods are goods that enter directly and these are ready for immediate consumption. Type- $b$ goods are capital goods in which the products of other capital goods enter directly and only the products of final goods enter indirectly.

- The economy is in a dynamic state, tending to increase capital equipment. A part of type- $a$ commodities is diverted from their normal destination and allocated to type $-b$.

- Let $\varepsilon_{1}$ and $\varepsilon_{2}$ denote positive constants. $\varepsilon_{1}$ shows the increase coefficient of type- $a$ goods and $-\varepsilon_{2}$ shows the increase coefficient of type- $b$ goods.

- The increase coefficient of type- $a$ goods through the type- $b$ goods is $-\gamma_{1}$ and the increase coefficient of type- $b$ goods through type- $a$ goods is $\gamma_{2}$. In here, $\gamma_{1}$ and $\gamma_{2}$ are positive constants.

Let consider $C_{1}$ and $C_{2}$ to show the volumes of type- $a$ and type- $b$ goods at time $t(t \geq 0)$. The model consisting of two nonlinear autonomous differential equation proposed by Palomba is

$$
\begin{aligned}
& \frac{d C_{1}}{d t}=C_{1}\left(\varepsilon_{1}-\gamma_{1} C_{2}\right)=\varepsilon_{1} C_{1}-\gamma_{1} C_{1} C_{2} \\
& \frac{d C_{2}}{d t}=-C_{2}\left(\varepsilon_{2}-\gamma_{2} C_{1}\right)=-\varepsilon_{2} C_{2}+\gamma_{2} C_{1} C_{2} .
\end{aligned}
$$

Palomba created his model using ordinary differential equations system and showed that a cyclical situation occurred in his analysis.

Daşbaşı and Boztosun [5] analyzed Palomba's model using incommensurate fractional-order differential equations system and explained the stability of equilibrium points according to different states of derivative orders.

According to the results of their model, there are followings:

- Trivial equilibrium point is always unstable.

- The positive equilibrium point is stable, when the sum of the derivative orders is between 0 and 2 .

- If the sum of the derivative orders is 2 or more, then the positive equilibrium point is unstable.

- They showed graphically the existence of cyclical state in case of instability.

In here, the Palomba model was analyzed using the difference equation system. Also, The nonmarketable loss rate of the first and second type goods were taken into account as parameters $\mu_{1}$ and $\mu_{2}$, respectively. Therefore, we have proposed the model,

$$
\begin{aligned}
& x_{t+1}=f\left(x_{t}, y_{t}\right)=x_{t}+\delta\left(x_{t}\left(\varepsilon_{1}-\gamma_{1} y_{t}-\mu_{1}\right)\right) \\
& y_{t+1}=g\left(x_{t}, y_{t}\right)=y_{t}-\delta\left(y_{t}\left(\varepsilon_{2}-\gamma_{2} x_{t}+\mu_{2}\right)\right)
\end{aligned}
$$

for $t \geq 0$ by means of the difference equations. In here, it is

$$
\varepsilon_{1}, \varepsilon_{2}, \gamma_{1}, \gamma_{2}, \delta, \mu_{1}, \mu_{2}>0
$$




\section{Existence and Stability of Fixed Point}

In this section, firstly, some definitions and theorems about the stability of equilibrium points of difference equation systems are given, then the fixed points (equilibrium points) of the system (2) were investigated and the stability of these points was analyzed.

Definition 2.1 That the equilibrium point of the first-order difference equation system given as

$$
X_{t+1}=F\left(X_{t}\right)
$$

is the point $\bar{X}$ that satisfies the equations $\bar{X}=F(\bar{X})$. Also, let us consider $J(\bar{X})$ to be the Jacobian matrix calculated at this equilibrium point. If the eigenvalues obtained from the equation $\operatorname{det}(J(\bar{X})-\lambda I)=0$ satisfy the conditions $\lambda_{i} \neq 1$ for $i=1,2, \ldots, n$, then this point is called hyperbolic equilibrium, otherwise it is called non-hyperbolic equilibrium [6].

Theorem 2.1 (Jury Conditions, Schur-Cohn Criterion) Considering the $n$-th degree characteristic equation of the system in (4) given as

$$
P(\lambda)=\lambda^{n}+a_{1} \lambda^{n-1}+a_{2} \lambda^{n-2}+\cdots+a_{n}
$$

it is presumed $\lambda_{i}$ for $i=1,2, \ldots, n$ as the eigenvalues obtained from the equation $P(\lambda)=0$. In this case, the local asymptotically stability (LAS) conditions of the equilibrium point are

$$
\left|\lambda_{i}\right|<1 \text {. }
$$

In addition, if the inequalities in Eq. (6) are satisfied, then we have followings:

- $P(1)=1+a_{1}+a_{2}+\cdots+a_{n}>0$,

- $(-1)^{n} P(-1)=1-a_{1}+a_{2}-\cdots+(-1)^{n} a_{n}>0$,

- $\left|a_{n}\right|<1 \quad$ [7].

Definition 2.2 Let's consider the difference equation system

$$
\begin{aligned}
& x_{t+1}=f\left(x_{t}, y_{t}\right) \\
& y_{t+1}=g\left(x_{t}, y_{t}\right)
\end{aligned}
$$

For this system, the point $(\bar{x}, \bar{y})$ that satisfies the condition

$$
\begin{aligned}
& \bar{x}=f(\bar{x}, \bar{y}) \\
& \bar{y}=g(\bar{x}, \bar{y}),
\end{aligned}
$$

is called as the equilibrium point [7].

Definition 2.3 Let us assumed that the functions $f(x, y)$ and $g(x, y)$ given in the system (7) have continuous partial derivatives for $x$ and $y$ on an open set of $\mathbb{R}_{+}^{2}$. Also, let $(\bar{x}, \bar{y})$ be the equilibrium point of the system and $\bar{J}=J(\bar{x}, \bar{y})=\left(\begin{array}{ll}f_{x}(\bar{x}, \bar{y}) & f_{y}(\bar{x}, \bar{y}) \\ g_{x}(\bar{x}, \bar{y}) & g_{y}(\bar{x}, \bar{y})\end{array}\right)$ be the Jacobian matrix at this point. The characteristic equation of the eigenvalues $\lambda_{i}$ for $i=1,2$ obtained from the equation $\operatorname{det}\left(J(\bar{X})-\lambda I_{2 \times 2}\right)=0$ is $[8]$

$$
\lambda^{2}-\operatorname{Tr}(\bar{J}) \lambda+\operatorname{Det}(\bar{J})=0
$$


Corollary 2.1 If the characteristic equation in Eq. (5) is taken into account for $n=2$, then

$$
P(\lambda)=\lambda^{2}+a_{1} \lambda+a_{2}
$$

is found. In here, it is $a_{1}=-\operatorname{Tr}(J)$ and $a_{2}=\operatorname{Det}(J)$. According to Theorem 2.1., if

$$
|\operatorname{Tr}(J)|<1+\operatorname{Det}(J)<2
$$

is satisfied, then the point $(\bar{x}, \bar{y})$ is LAS [9].

Proposition 2.1 The system (2) have

- the trivial equilibrium point $E_{0}(0,0)$, and

- the positive equilibrium point $E_{1}\left(\frac{\left(\varepsilon_{2}+\mu_{2}\right)}{\gamma_{2}}, \frac{\left(\varepsilon_{1}-\mu_{1}\right)}{\gamma_{1}}\right)$, when $\left(\varepsilon_{1}-\mu_{1}\right)>0$.

Proposition 2.2 The system (2) has two equilibrium points with the following dynamical properties:

- the trivial equilibrium point $E_{0}(0,0)$ is LAS, when $\left(\varepsilon_{1}-\mu_{1}\right)<0$.

- let $\left(\varepsilon_{1}-\mu_{1}\right)>0$. the nontrivial equilibrium point $E_{1}\left(\frac{\left(\varepsilon_{2}+\mu_{2}\right)}{\gamma_{2}}, \frac{\left(\varepsilon_{1}-\mu_{1}\right)}{\gamma_{1}}\right)$ is always unstable point.

Proof The Jacobian matrix of (2) is

$$
J(x, y)=\left(\begin{array}{cc}
1+\delta\left(\left(\varepsilon_{1}-\mu_{1}\right)-\gamma_{1} y\right) & -\delta \gamma_{1} x \\
\delta \gamma_{2} y & 1-\delta\left(\left(\varepsilon_{2}+\mu_{2}\right)-\gamma_{2} x\right)
\end{array}\right)
$$

through of partial derivatives.

- The Jacobian matrix in Eq. (12) calculated at the equilibrium point $E_{0}(0,0)$ is $J\left(E_{0}(0,0)\right)=\left(\begin{array}{cc}\left(1+\delta\left(\varepsilon_{1}-\mu_{1}\right)\right) & 0 \\ 0 & \left(1-\delta\left(\varepsilon_{2}+\mu_{2}\right)\right)\end{array}\right)$. In addition, the characteristic equation for this point is

$$
\lambda^{2}-\left(\left(1+\delta\left(\varepsilon_{1}-\mu_{1}\right)\right)+\left(1-\delta\left(\varepsilon_{2}+\mu_{2}\right)\right)\right) \lambda+\left(1+\delta\left(\varepsilon_{1}-\mu_{1}\right)\right)\left(1-\delta\left(\varepsilon_{2}+\mu_{2}\right)\right)=0
$$

and the eigenvalues are found as $\lambda_{1}=\left(1+\delta\left(\varepsilon_{1}-\mu_{1}\right)\right)$ and $\lambda_{2}=\left(1-\delta\left(\varepsilon_{2}+\mu_{2}\right)\right)$. It is obvious that the eigenvalues are positive real numbers due to Eq. (3). According to the stability conditions of the equilibrium point in Eq. (6), if

$$
\begin{gathered}
-\frac{2}{\delta}<\left(\varepsilon_{1}-\mu_{1}\right)<0 \\
\left(\varepsilon_{2}+\mu_{2}\right)<\frac{2}{\delta}
\end{gathered}
$$


then it is $\left|\lambda_{1}\right|<1$ and $\left|\lambda_{2}\right|<1$. Consequently, $E_{0}$ is LAS. When the inequalities in (14a) and (14b) are evaluated together, the stability condition of this point can be considered as

$$
0<\left\{\left(\mu_{1}-\varepsilon_{1}\right) \text { and }\left(\mu_{2}+\varepsilon_{2}\right)\right\}<\frac{2}{\delta}
$$

- Let $\left(\varepsilon_{1}-\mu_{1}\right)>0$. In this case, $E_{1}$ exists. The Jacobian matrix evaluated at $E_{1}\left(\frac{\left(\varepsilon_{2}+\mu_{2}\right)}{\gamma_{2}}, \frac{\left(\varepsilon_{1}-\mu_{1}\right)}{\gamma_{1}}\right)$ becomes $J\left(E_{1}\right)=\left(\begin{array}{cc}1 & -\delta \gamma_{1} \frac{\left(\varepsilon_{2}+\mu_{2}\right)}{\gamma_{2}} \\ \delta \gamma_{2} \frac{\left(\varepsilon_{1}-\mu_{1}\right)}{\gamma_{1}} & 1\end{array}\right)$ and thus the characteristic equation is

$$
\lambda^{2}-2 \lambda+\left(1+\delta^{2}\left(\varepsilon_{1}-\mu_{1}\right)\left(\varepsilon_{2}+\mu_{2}\right)\right)=0
$$

According to Corollary 2.1 , the stability conditions are not met. Therefore, $E_{1}$ is unstable point. Proof is completed. As a result of the stability analysis, the following Table can be reached.

Table 1. Existence and LAS conditions of equilibrium points of system (2)

\begin{tabular}{ccc}
\hline Equilibrium point & Existence condition & LAS condition \\
\hline$E_{0}(0,0)$ & Always exists & If $0<\left\{\left(\mu_{1}-\varepsilon_{1}\right)\right.$ and $\left.\left(\mu_{2}+\varepsilon_{2}\right)\right\}<\frac{2}{\delta}$ \\
$E_{1}\left(\frac{\left(\varepsilon_{2}+\mu_{2}\right)}{\gamma_{2}}, \frac{\left(\varepsilon_{1}-\mu_{1}\right)}{\gamma_{1}}\right)$ & $\left(\varepsilon_{1}-\mu_{1}\right)>0$ & Unstable \\
\hline
\end{tabular}

Corollary 2.2 Let $\varepsilon_{1}=\mu_{1}$. By Eq. (16), characteristic equation is

$$
\lambda^{2}-2 \lambda+1=0 .
$$

Therefore, the eigenvalues are $\lambda_{1}=\lambda_{2}=1$, and so, $E_{1}\left(\frac{\left(\varepsilon_{2}+\mu_{2}\right)}{\gamma_{2}}, 0\right)$ hyperbolic equilibrium point.

Corollary 2.3 $E_{0}$ is unstable point, when $E_{1}$ exists. Therefore, these equilibrium points can not be stable at together. This situation is also seen in the Table 1.

\section{Numerical Studies}

In this section, the system (2) is graphically shown by giving the values to the parameters used in the system to support the qualitative analysis results shown in Table 1 . These values are shown in Table 2. 
Table 2. The considered values of the parameters.

\begin{tabular}{ccc}
\hline \multirow{2}{*}{ Parameters } & \multicolumn{2}{c}{ Values } \\
& For Fig. 1 & For Fig. 2 \\
\hline$\delta$ & .4 & .4 \\
$\varepsilon_{1}$ & .9 & 1.1 \\
$\gamma_{1}$ & .01 & .01 \\
$\mu_{1}$ & 1 & 1 \\
$\varepsilon_{2}$ & .1 & .1 \\
$\gamma_{2}$ & .1 & .1 \\
$\mu_{2}$ & .5 & .5 \\
Initial conditions & $x(1)=3$ and $y(1)=2$ \\
\hline
\end{tabular}

From first column values in Table $2, E_{1}$ is not exists due to $\left(\varepsilon_{1}-\mu_{1}\right)=.9-1=-.1<0$. It is clear that $0<\{\overbrace{\left(\mu_{1}-\varepsilon_{1}\right)}^{.1}$ and $\overbrace{\left(\mu_{2}+\varepsilon_{2}\right)}^{.6}\}<\frac{{ }^{2}}{\delta}$ in Eq. (15) is satisfied. Therefore, the trivial equilibrium point $E_{0}(0,0)$ is LAS. Fig. 1 shows this situation.
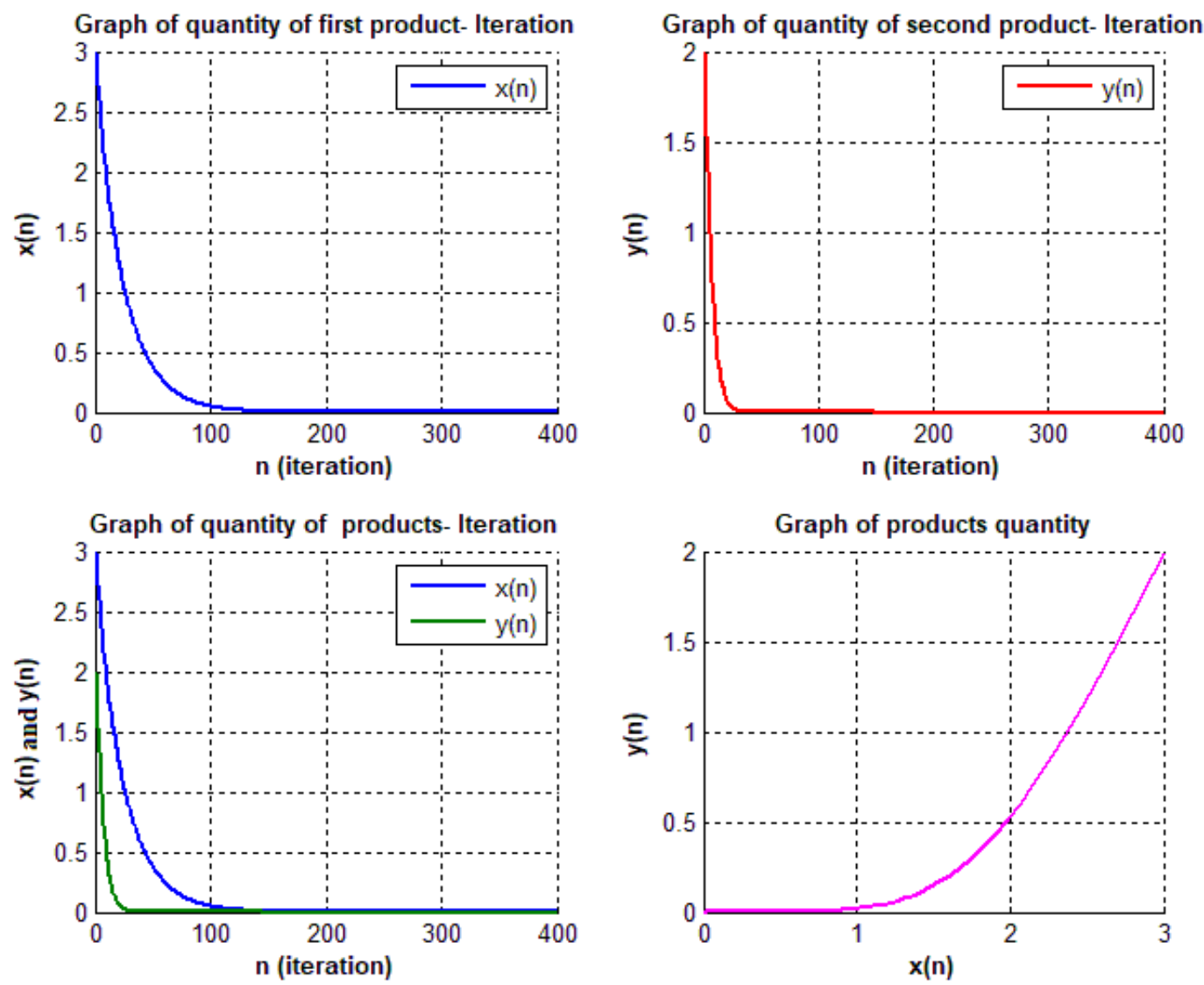

Fig. 1. For first column values in Table 2., time-dependent changes in quantities of the first and second products 
From second column values in Table 2 , the nontrivial equilibrium point is found as $E_{1}(6,10)$. Inequalities in Eq. (15) is not satisfied. Therefore, $E_{0}$ is unstable point. The existence of periodic orbits can be seen in Fig. 2.
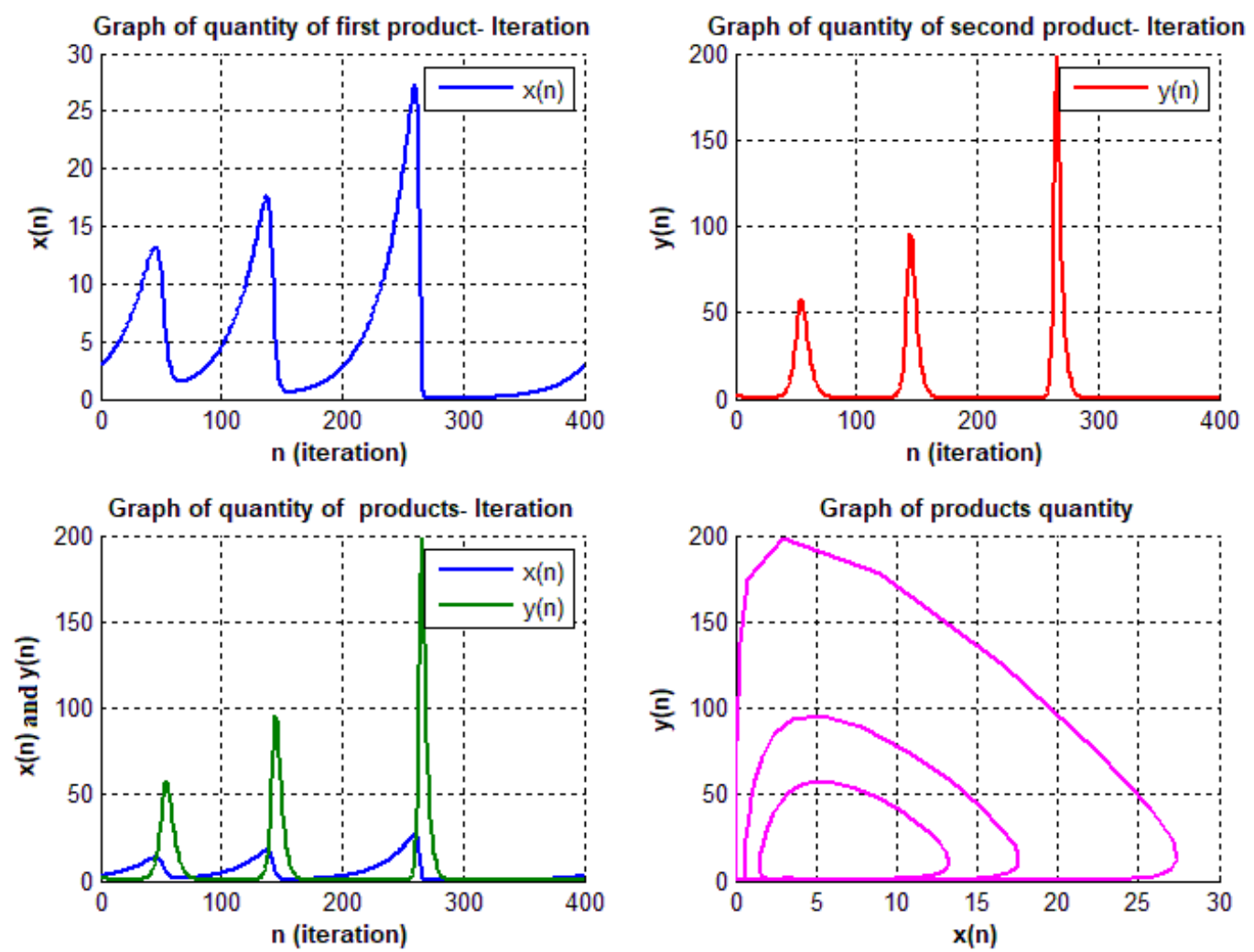

Fig.2. For second column values in Table 2, time-dependent changes in quantities of the first and second products

\section{Result and Discussion}

Palomba was the first to use the Lotka-Volterra equations. According to the results of the analysis of the model where he proposed through the ordinary differential equations, he explained that the volumes of goods will be in a cyclical state [4].

Daşbaşı and Boztosun [5] studied the Palomba model using incommensurate fractional-order differential equation system in Caputo meaning. They showed the order of derivatives in the equations expressing the first and second type goods with $\alpha_{1}$ and $\alpha_{2}$, respectively. Also, they expressed the existence and stability of the fixed points of the system. In the case of $0<$ $\alpha_{1}, \alpha_{2}<1$, the volumes of these products approach positive values depending on time. Contrary to the cyclical situation, stability analysis of the positive equilibrium point under certain conditions was made and shown with graphs.

In here, the Palomba economic model was modeled by difference equation systems and then analyzed. In addition, the followings were taken into account in the model: the non-marketable 
loss rate of the first and second type goods are parameters $\mu_{1}$ and $\mu_{2}$, respectively. Trivial and positive (Non-trivial) equilibrium points were found in the analysis. The stability of the trivial equilibrium point under certain conditions has been expressed. Although the positive equilibrium point exists under certain conditions, it has been shown to be an unstable point. Therefore, it can be said that there is a cyclical situation in harmony with Palomba.

\section{References}

[1] Daşbaşı, B., Daşbaşı, T., Doğrusal Programlama Minimizasyon Problemi İçin Matematiksel Model Ve Uygulamas1: Kimyasal Gübre Alım1, The Journal of International Social Research, 10(50), 675-683, 2017.

[2] Mondal, S.P., Khan, N.A., Vishwakarma, D., Saha, A.K., Existence and Stability of Difference Equation in Imprecise Enviroment, Nonlinear Engineering, 7(4), 263-271, 2018.

[3] Harvie, D., Kelmanson, M.A., Knapp, D.G., A Dynamical Model of Business Cycle Asymmetries: Extending Goodwin, Economic, 12, 53-92, 2007.

[4] Gandolfo, G., Giuseppe Palomba and the Lotka-Volterra Equations, Rendiconti Lincei, 19(4), 347-357, 2008.

[5] Daşbaşı, B., Boztosun, D., Çoklu Kesirli Mertebeden Diferansiyel Denklemler ile Palomba Ekonomi Modelinin Kararlılık Analizi, Journal of International Social Research, 11(59), 901-907, 2018.

[6] Elaydi, S.N., An Introduction To Difference Equations, Third Edition ed., Springer, 2005.

[7] Allen, L.J.S., An Introduction to Mathematical Biology., 2007, ISBN 10: 0-13-035216-0.

[8] Fulford, G., Forrester, P., Jones, A., Modelling with Differential and Difference Equations (Australian Mathematical Society Lecture Series Book 10), 1st ed. U.K., Cambridge University Press, 2001 (Reprinted).

[9] Mickens, R.E., Difference equations: theory, applications and advanced topics, Third Edition ed., CRC Press, 2015. 